\title{
Development and Validation of HPLC-PDA Method and Pattern Recognition Analyses Using Eight Marker Compounds for the Quality Control Between the Seeds of Cuscuta chinensis Lam. and Cuscuta japonica Choisy
}

\author{
Duc Hung Nguyen ${ }^{1,2}$, Bing Tian Zhao ${ }^{1,3}$, Duc Dat Le ${ }^{1,4,5}$, Eun Sook Ma ${ }^{1}$, Byung Sun Min ${ }^{1}$, and Mi Hee Woo ${ }^{1, *}$ \\ ${ }^{1}$ College of Pharmacy, Drug Research and Development Center, Daegu Catholic University, \\ Gyeongsan 38430, Republic of Korea \\ ${ }^{2}$ Faculty of Medicine and Pharmacy, Thanh Dong University, Hai Duong city, Viet Na. \\ ${ }^{3}$ School of Chemical and Material Engineering, Jiangnan University, Wuxi 214122, China \\ ${ }^{4}$ Division of Computational Physics, Institute for Computational Science, Ton Duc Thang University, Ho Chi Minh City, Vietnam. \\ ${ }^{5}$ Faculty of Pharmacy, Ton Duc Thang University, Ho Chi Minh City, Vietnam.
}

\begin{abstract}
Cuscuta chinensis Lam. and Cuscuta japonica Choisy are parasitic plants. C. chinensis seeds were traditionally used for treatment of kidney and liver deficiencies. C. japonica seeds were used as tonic medicine to improve liver function and strengthen kidneys, treatment of high blood pressure, chronic diarrhea, and sore eyes. Cuscutae Semen are seeds of only $C$. chinensis in Korean Herbal Pharmacopoeia (K.H.P.). The developed HPLC-PDA method easily, accurately, and sensitively quantified using eight marker compounds [hyperoside (1), astragalin, (2), quercetin (3), kaempferol (4), chlorogenic acid (5), 3,4-di-O-caffeoylquinic acid (6), 1,5-di-Ocaffeoylquinic acid (7), and 4,5-di-O-caffeoylquinic acid (8)]. In addition, the method may be used to distinguish seeds between $C$. chinensis Lam. and $C$. japonica Choisy. Furthermore, the result from the current study was applied to clarify samples between steam processed and unprocessed samples of $C$. chinensis by pattern analysis. Keywords - Cuscuta chinensis, Cuscuta japonica, Flavonoids, Caffeoylquinic acid, HPLC-PDA
\end{abstract}

\section{Introduction}

Cuscuta chinensis Lam. is a parasitic plant belonging to the Convolvulaceae family. It is widely distributed in Korea, China, Japan, and Africa. ${ }^{1}$ Stems are yellow and thin. Seeds were pale brown, ovoid, scabrous, and diameter of 1-3 mm. It was traditionally used as a medicine for the treatment of kidney and liver deficiency. ${ }^{2}$ In addition, pastes of this plant are used to treat chronic ulcer, wounds, painful inflammation, sore head, and inflamed eyes. ${ }^{1}$ In Korea and Vietnam, seeds of $C$. chinensis are applied for sexual function, health, and back pain. ${ }^{3}$ Several studies reported that $C$. chinensis possesses various biological activities such as hepatoprotective, antiosteoporotic, ${ }^{4}$ neuroprotection, ${ }^{5}$ antioxidant, ${ }^{6}$ anti-aging, ${ }^{7}$ anti-cancer, and anti-diabetic activities. ${ }^{8}$ Chemical constituents of $C$. chinensis are reported as flavonoids, alkaloids, steroids, fatty acid, volatile oils, lignans, quinic acid

\footnotetext{
*Author for correspondence

Mi Hee Woo, College of Pharmacy, Drug Research and Development Center, Daegu Catholic University, Gyeongsan 38430, Republic of Korea

Tel: +82-53-850-3620; Email: woomh@cu.ac.kr
}

derivatives and polysaccharides. ${ }^{9}$ Flavonoids, a large group in natural products, reveal various pharmacological activities. Some flavonoids in this plant such as kaempferol, hyperoside, astragalin, and quercetin relate to the mechanism of clinical effects. Therefore, main flavonoid components in this plant would be interesting in determining its quality evaluation.

C. japonica Choisy is a typical dodder with yellowish vines or purplish spots, lightly tout. Seeds were brown with ovoid capsule and $3-5 \mathrm{~mm}$ in diameter. They were used as tonic medicine to improve liver function and strengthen kidneys, treatment of high blood pressure, chronic diarrhea, and sore eyes. In the Korean herb market, there are confused uses of seeds of $C$. japonica as Cuscutae Semen because it is difficult to distinguish dried seed specimens between $C$. chinensis and $C$. japonica. However, Cuscutae Semen in K.H.P. are seeds of only $C$. chinensis. Different species, environmental conditions, and locations lead to differences in chemical constituents and pharmacological properties. ${ }^{10}$ Therefore, an effective characterization method for quality control of seeds between $C$. chinensis and $C$. japonica is desirable. Until 
now, there are few studies on quantitative constituents in $C$. chinensis as well as C. japonica. Therefore, in this study, we describe development of the analysis method to simultaneously determine eight marker compounds with simple, rapid, and accurate analysis by reversed phase liquid chromatography in $C$. chinensis and $C$. japonica samples.

\section{Experimental}

Chemicals and reagents - Unless specified, all reagents used were of analytical grade. The standards were purchased from Sigma-Adrich Chemical Co. (St. Louis, MO, USA). The standard compound structures are shown in Fig. 1. Acetonitrile (ACN) was purchased from Fisher Scientific Korea LtD. Water was purified using a Milli-Q system (Millipore, Bedford, USA).

Plant material collection - The seeds of $C$. chinensis (C01 to C06) and C. japonica (J07 to J12) were identified and authenticated by Prof. Byung Sun Min. The voucher specimens (C.2017001 to C.2017012) of the samples were deposited in Herbarium at College of Pharmacy,
Daegu Catholic University, Korea.

Standard solution-Standard stock solutions were prepared separately for each analytical standard and an internal standard (IS) in $2 \mathrm{~mL} \mathrm{MeOH}$ at $1000 \mu \mathrm{g} / \mathrm{mL}$ and diluted with $\mathrm{MeOH}$ to obtain appropriate concentrations for content determination. The solutions were transferred to $10 \mathrm{~mL}$ amber glass vials, sealed using elastic plastic film (Parafilm, Chicago, IL, USA) and stored in a refrigerator $\left(4^{\circ} \mathrm{C}\right)$ for analysis.

Sample preparation - The dried seeds of $C$. chinensis was pulverized and passed through a 180 mesh sieve. About $1 \mathrm{~g}$ of the powder, accurately weighed, were added to $40 \mathrm{~mL}$ of $75 \%$ methanol containing an internal standard (IS: $20 \mathrm{ppm}$ caffeic acid) by sonication for 60 minutes. After extraction, each sample solution was adjusted to the original volume and filtered through $0.45 \mu \mathrm{m}$ membrane, and an aliquot $(10 \mu \mathrm{L})$ of the filtrate was injected into HPLC.

HPLC analyses - The HPLC experiments were performed using a Waters Alliance system (Waters, Houston, TX, USA) equipped with a vacuum degasser, PDA detector and an Aegispak C18-L $(250 \mathrm{~mm} \times 4.6 \mathrm{~mm}, 5$<smiles>O=C1OC(Oc2c(-c3ccc(O)c(O)c3)oc3cc(O)cc(O)c3c2=O)C(O)C(O)C(O)C1O</smiles>

1<smiles></smiles>

4<smiles>O=C1OC(Oc2c(-c3ccc(O)cc3)oc3cc(O)cc(O)c3c2=O)C(O)C(O)C1O</smiles>

2<smiles></smiles>

3<smiles>C=CC(=O)O[C@@H]1C[C@@](O)(C(=O)O)[C@H](O)C[C@H]1O</smiles>

5<smiles>O=C(O)C1C[C@](O)(C(=O)O)C[C@H](O)[C@H]1O</smiles>

6

Fig. 1. The structures of marker compounds $(\mathbf{1}-\mathbf{8})$ of $C$. chinensis and $C$. japonica samples. 
$\mu \mathrm{m})$ column. Data handling was managed by Empower v.3.0 software.

HPLC instrument and chromatographic conditions HPLC-PDA analyses were performed on a Waters (Houston, TX, USA) equipped with an autosampler, degasser, quaternary solvent pump, and PDA detector (Waters 2998) scanning in the wavelength range of 190 $400 \mathrm{~nm}$. Separation was carried out on an Aegispak C18L column $(4.6 \times 250 \mathrm{~mm}, 5 \mu \mathrm{m}$ particle size; Young JinBiochrom, Korea). UV detection was recorded at the wavelengths of $349 \mathrm{~nm}$. The mobile phase consisted of solvent $\mathrm{A}\left(\mathrm{H}_{2} \mathrm{O}\right.$ containing $0.3 \%$ formic acid $)$ and solvent $\mathrm{B}$ (acetonitrile), and gradient elution profile was conducted as follows: 0 - $30 \mathrm{~min}, 17 \% \mathrm{~B} ; 30-50 \mathrm{~min}, 17-55 \% \mathrm{~B}$. The flow rate for mobile phase was set at $1 \mathrm{~mL} / \mathrm{min}$ and the injection volumes were $10 \mu \mathrm{L}$.

Method validation - The analytical method for the seeds of C. chinensis was validated by the determination of linearity, limit of detection (LOD) and limit of quantitation (LOQ), accuracy, precision, stability, and robustness. $^{11}$

Linearity - Eight marker compounds were accurately weighed and dissolved in methanol $1000 \mu \mathrm{g} / \mathrm{mL}$ as stock solutions. The stock solutions were then diluted to produce different concentrations for each marker. Linearity was determined by plotting the measurements of area peak ratios (analyte/IS) versus concentrations of analytical standards. The sensitivity was expressed by the LOD and LOQ. The LOD represents the lowest concentration that can be reliably determined at a signal-to-noise $(\mathrm{S} / \mathrm{N})$ ratio of 3. The estimate for the LOQ was calculated using $\mathrm{S} / \mathrm{N}$ ratio of 10 .

Accuracy and Precision - Intra-day $(n=5)$ and interday $(n=5)$ precisions and accuracies were evaluated by analyzing sets of five independent samples at the low, mid, and high concentration levels. The precision was expressed as RSD\% and the accuracy was expressed as bias. The stability of marker compounds was analyzed by the sample solution of aerial parts of $C$. chinensis through storing extract solution in the dark at $4{ }^{\circ} \mathrm{C}$ and at room temperature $\left(25^{\circ} \mathrm{C}\right)$. The two samples were analyzed in triplicate at $0,1,3,7,15$, and 30 days separation. ${ }^{12}$

Pattern recognition analysis - The determination of the phytotaxonomic or phytochemical relationship of 12 samples [six C. chinensis Lam. (C01-C06) and six C. japonica Choisy (J07-J12)], pattern recognition analysis was conducted using software IBM SPSS Statistics Version 22.

Optimized method - A gradient RP-C18 HPLC system was performed for the simultaneous quantitative determination of eight compounds: hyperoside (1), astragalin, (2), quercetin (3), kaempferol (4), chlorogenic acid (5), 3,4-di- $O$-caffeoylquinic acid (6), 1,5-di- $O$ caffeoylquinic acid (7), and 4,5-di- $O$-caffeoylquinic acid (8) in extracts from $C$. chinensis and $C$. japonica seeds. These compounds showed a high UV absorption at 360 $\mathrm{nm}$, therefore this wavelength was used for their quantitative determination. Mixtures of $\mathrm{ACN}$ (B) and water containing $0.3 \%$ formic acid (A) were examined as the mobile phases, with different ratios as well as a gradient elution system were optimized. The most suitable gradient elution system was 0 - $30 \mathrm{~min}, 17 \%$ (B); 30 - $50 \mathrm{~min}, 17$ $55 \%$ (B), v/v. The chromatographic peaks of the markers in the sample solution were determined by comparing their retention times with those of the individual standards, and were confirmed by spiking the samples with the individual compounds. Compounds $(\mathbf{1}-\mathbf{4})$ appeared in the chromatograms of $C$. chinensis samples, meanwhile compound (5-8) appeared in those of $C$. japonica samples (Fig. 2). The resolutions of all marker compounds in this chromatogram are much better and clearer than the previous report. $^{13}$

Optimized extract - Extraction process was optimized by using methanol-water and ethanol-water with different ratios. In this study, the seeds of $C$. chinensis and $C$. japonica were grinded for $5 \mathrm{~min}$ and were then sieved through a $250 \mu^{2}$ sieve. $^{12}$ The various solvent systems of ethanol-water mixtures $(95 \%, 75 \%, 50 \%, 25 \%)$ and methanol-water $(100 \%, 75 \%, 50 \%, 25 \%)$ were used to analysis of the $C$. chinensis and $C$. japonica samples to maximize recovery of all markers. The $C$. chinensis and C. japonica samples (each sample, $1.0 \mathrm{~g}$ ) were extracted with $40 \mathrm{~mL}$ of above solvents for $60 \mathrm{~min}$ at room temperature in an ultrasonic bath. As a result, 75\% aqueous methanol was selected as extract solvent system for $C$. chinensis and $C$. japonica samples due to the greatest peak areas of the markers $\mathbf{1 - 4}$ and $\mathbf{5 - 8}$ in their extraction, respectively. Similarly, the ultra-sonication was selected due to their higher area peaks in ultra-sonication than those of the reflux. To evaluate the optimized extraction times, five period times $(30,45,60,75$, and 90 min) were examined in $75 \%$ methanol via sonication at room temperature. The results showed that each area peak of analytes was not increased from $60 \mathrm{~min}$. Therefore, the extraction time was optimized as $60 \mathrm{~min}$.

Analytical method validation - The linearity was evaluated by using seven different concentrations of each analyte $(0.625,6.25,12.5,25,50,100$, and $200 \mu \mathrm{g} / \mathrm{mL})$ for $C$. chinensis sample and $(0.625,6.25,12.5,25,50$, 100,200 , and $500 \mu \mathrm{g} / \mathrm{mL}$ ) for $C$. japonica sample and in 

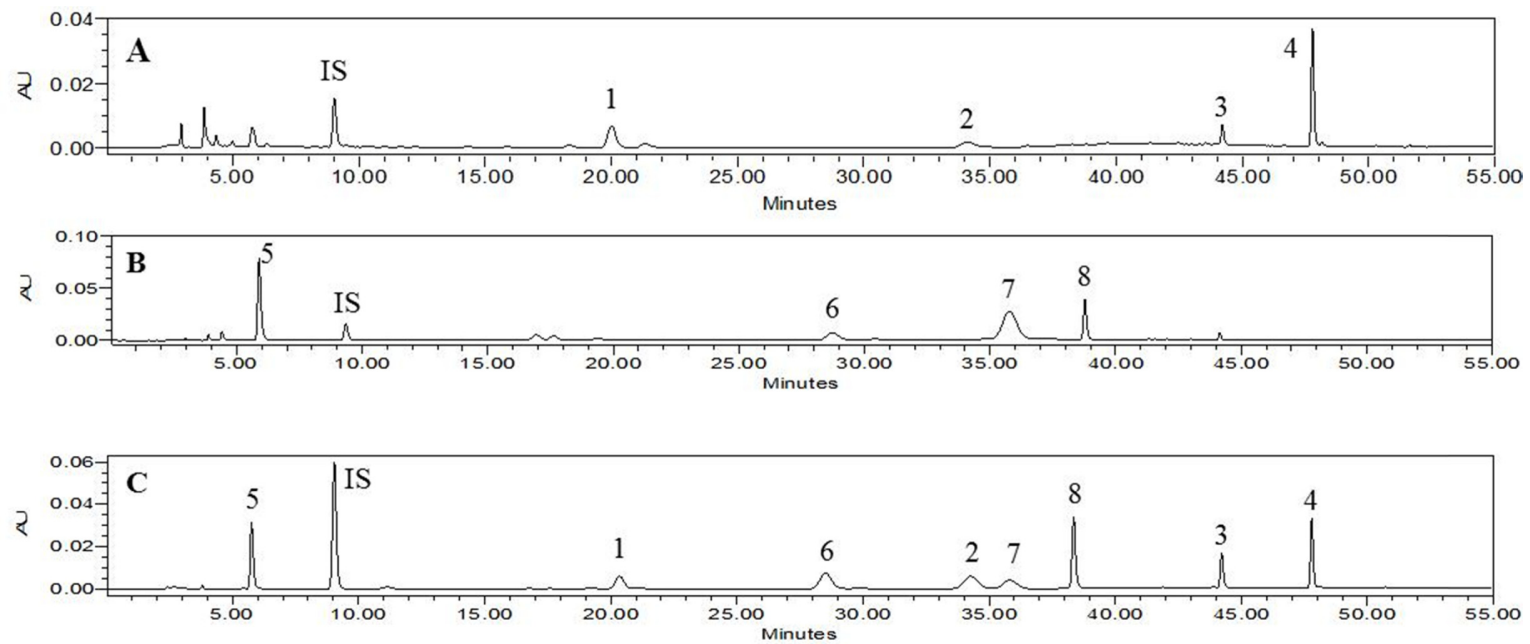

Fig. 2. The HPLC chromatograms of $C$. chinensis (A) and $C$. japonica (B) samples and the standard mixture (C). Hyperoside (1), astragalin (2), quercetin (3), kaempferol (4), chlorogenic acid (5), 3,4-di-O-caffeoylquinic acid (6), 1,5-di-O-caffeoylquinic acid (7), 4,5$\mathrm{di}-\mathrm{O}$-caffeoylquinic acid (8), and an internal standard (IS, caffeic acid).

Table 1. The linearity, linear range, LOD, and LOQ

\begin{tabular}{ccccccc}
\hline \hline Analytes & $\begin{array}{c}\text { Linear range } \\
(\mu \mathrm{g} / \mathrm{mL})\end{array}$ & Slope & Intercept & $\begin{array}{c}\text { Correlation } \\
\text { coefficient }\left(r^{2}\right)\end{array}$ & $\begin{array}{c}\text { LOD } \\
(\mu \mathrm{g} / \mathrm{mL})\end{array}$ & $\begin{array}{c}\text { LOQ } \\
(\mu \mathrm{g} / \mathrm{mL})\end{array}$ \\
\hline $\mathbf{1}$ & $0.625-200$ & 0.0931 & 0.3222 & 0.9991 & 0.015 & 0.051 \\
$\mathbf{2}$ & $0.625-200$ & 0.0705 & 0.1806 & 0.9991 & 0.020 & 0.069 \\
$\mathbf{3}$ & $0.625-200$ & 0.1181 & 0.2140 & 0.9994 & 0.013 & 0.043 \\
$\mathbf{4}$ & $0.625-200$ & 0.1957 & 0.2824 & 0.9998 & 0.006 & 0.023 \\
$\mathbf{5}$ & $0.625-500$ & 0.0297 & 0.0750 & 0.9994 & 0.057 & 0.190 \\
$\mathbf{6}$ & $0.625-500$ & 0.0301 & 0.0763 & 0.9994 & 0.086 & 0.288 \\
$\mathbf{7}$ & $0.625-500$ & 0.0381 & 0.0594 & 0.9996 & 0.045 & 0.151 \\
$\mathbf{8}$ & $0.625-500$ & 0.0585 & 0.1204 & 0.9991 & 0.031 & 0.104 \\
\hline
\end{tabular}

triplicate analysis. The calibration curves of each analyte showed excellent linearity over the tested range $\left(r^{2}>\right.$ 0.9991) (Table 1). The LOD of each analyte was determined to be 0.006 to $0.086 \mu \mathrm{g} / \mathrm{mL}$ and the $\mathrm{LOQ}$ was 0.023 to $0.288 \mu \mathrm{g} / \mathrm{mL}$ indicating that the developed method for the seeds of $C$. chinensis and $C$. japonica exhibited good sensitivity for determination of these marker compounds. The accuracy of the developed HPLC method was determined by analyzing the known amounts at the three different concentrations (each analyte: 1, 50, and $200 \mu \mathrm{g} / \mathrm{mL}$ ) of analytes spiked into $75 \%$ methanol extract solution of the seeds of $C$. chinensis (marker compounds: 1 - 4) and C. japonica (marker compounds: 5 - 8). After addition of known amounts of each analyte to the previous $75 \%$ methanol extract solution, recovery studies were carried out. The results were listed in Table 2. In C. chinensis and C. japonica samples, the precisions for marker compounds (1 - 8) were less than $12.61 \%$ in intra-day and $13.25 \%$ in inter-day. The accuracies of the method were in the range $87.82-107.48 \%$ in intra-day and $87.02-111.13 \%$ in inter-day. With above data, the method developed was precise, accurate, and reliable for quantitation analysis of the two species $(C$. chinensis and C. japonica).

Stability of marker compounds - To evaluate the stability of the analytes, the $75 \%$ methanol extract solution of $C$. chinensis and $C$. japonica samples at room temperature $\left(25^{\circ} \mathrm{C}\right)$ and $4{ }^{\circ} \mathrm{C}$ were measured at $0,1,3,7$, 15 , and 30 days. As the results, the marker compounds (1 - 8) showed stable with recovery ranging from 98.1 to $101.6 \%$ (Table 3).

Quantitation of eight marker compounds $(1-8)$ in $75 \%$ methanol extract of $C$. chinensis Lam. and $C$. japonica Choisy - The contents of each analyte present in each sample $(C$. chinensis and $C$. japonica) were listed in Table 4, in which C01-C03 samples of C. chinensis samples were steam processed. Two main analytes, compounds 1 and 4, were found in C. chinensis samples 
Table 2. Intra- and inter-day precisions of the eight marker compounds in $75 \% \mathrm{MeOH}$ extract of C. chinensis (1 - 4) and C. japonica (5 - 8)

\begin{tabular}{|c|c|c|c|c|c|c|c|c|c|c|c|}
\hline \multirow[b]{2}{*}{ Analyte } & \multirow{2}{*}{$\begin{array}{l}\text { Fortified } \\
\text { conc. } \\
(\mu \mathrm{g} / \mathrm{mL})\end{array}$} & \multirow{2}{*}{$\begin{array}{l}\text { Sample } \\
\text { conc. } \\
(\mu \mathrm{g} / \mathrm{mL})\end{array}$} & \multicolumn{4}{|c|}{ Intra-day $(n=5)$} & \multirow{2}{*}{$\begin{array}{l}\text { Sample } \\
\text { conc. } \\
(\mu \mathrm{g} / \mathrm{mL})\end{array}$} & \multicolumn{4}{|c|}{ Inter-day $(n=5)$} \\
\hline & & & $\begin{array}{c}\text { Observed } \\
(\mu \mathrm{g} / \mathrm{mL})\end{array}$ & SD & $\begin{array}{c}\text { Accuracy } \\
(\%)\end{array}$ & $\begin{array}{c}\text { Precision } \\
(\%)\end{array}$ & & $\begin{array}{c}\text { Observed } \\
(\mu \mathrm{g} / \mathrm{mL})\end{array}$ & SD & $\begin{array}{c}\text { Accuracy } \\
(\%)\end{array}$ & $\begin{array}{c}\text { Precision } \\
(\%)\end{array}$ \\
\hline \multirow{3}{*}{1} & 1 & 7.39 & 8.47 & 0.15 & 107.48 & 7.30 & 7.39 & 8.42 & 0.11 & 103.63 & 10.96 \\
\hline & 50 & 7.39 & 58.18 & 0.80 & 101.58 & 1.61 & 7.39 & 57.55 & 1.01 & 100.33 & 2.04 \\
\hline & 200 & 7.39 & 208.53 & 1.92 & 100.57 & 0.96 & 7.39 & 209.89 & 2.23 & 101.25 & 1.11 \\
\hline \multirow{3}{*}{2} & 1 & 3.72 & 4.70 & 0.15 & 97.85 & 5.36 & 3.72 & 4.81 & 0.10 & 109.10 & 8.35 \\
\hline & 50 & 3.72 & 53.20 & 0.56 & 98.95 & 1.12 & 3.72 & 53.06 & 0.30 & 98.67 & 0.60 \\
\hline & 200 & 3.72 & 200.20 & 2.81 & 98.24 & 1.12 & 3.72 & 203.64 & 2.21 & 99.96 & 1.11 \\
\hline \multirow{3}{*}{3} & 1 & 1.70 & 2.56 & 0.14 & 96.00 & 10.96 & 1.70 & 2.53 & 0.12 & 87.02 & 12.64 \\
\hline & 50 & 1.70 & 51.65 & 2.16 & 99.90 & 4.32 & 1.70 & 52.36 & 1.59 & 101.40 & 3.18 \\
\hline & 200 & 1.70 & 201.62 & 2.54 & 99.65 & 1.27 & 1.70 & 200.84 & 2.72 & 99.59 & 1.35 \\
\hline \multirow{3}{*}{4} & 1 & 7.15 & 8.37 & 0.23 & 89.16 & 10.96 & 7.15 & 8.23 & 0.18 & 107.66 & 13.25 \\
\hline & 50 & 7.15 & 53.11 & 2.23 & 99.77 & 4.45 & 7.15 & 57.24 & 1.39 & 100.17 & 2.78 \\
\hline & 200 & 7.15 & 201.85 & 3.47 & 99.54 & 1.73 & 7.15 & 208.33 & 1.40 & 100.59 & 0.70 \\
\hline \multirow{3}{*}{5} & 1 & 95.11 & 96.10 & 0.13 & 99.54 & 11.59 & 95.11 & 96.05 & 0.18 & 94.24 & 12.19 \\
\hline & 50 & 95.11 & 144.72 & 1.49 & 99.22 & 2.99 & 95.11 & 145.05 & 1.46 & 99.87 & 2.91 \\
\hline & 200 & 95.11 & 296.58 & 1.55 & 100.73 & 0.77 & 95.11 & 296.18 & 1.53 & 100.53 & 0.76 \\
\hline \multirow{3}{*}{6} & 1 & 10.78 & 11.66 & 0.19 & 87.82 & 12.61 & 10.78 & 11.80 & 0.12 & 101.57 & 11.71 \\
\hline & 50 & 10.78 & 60.71 & 1.23 & 99.86 & 2.47 & 10.78 & 59.88 & 0.78 & 98.19 & 1.55 \\
\hline & 200 & 10.78 & 211.44 & 3.23 & 100.33 & 1.61 & 10.78 & 210.51 & 4.78 & 99.86 & 2.38 \\
\hline \multirow{3}{*}{7} & 1 & 120.32 & 121.56 & 0.08 & 98.52 & 8.47 & 120.32 & 121.66 & 0.27 & 104.37 & 2.11 \\
\hline & 50 & 120.32 & 171.10 & 1.81 & 101.56 & 3.62 & 120.32 & 170.72 & 1.77 & 100.79 & 3.53 \\
\hline & 200 & 120.32 & 320.95 & 1.43 & 100.31 & 0.71 & 120.32 & 321.31 & 2.07 & 100.49 & 1.03 \\
\hline \multirow{3}{*}{8} & 1 & 13.80 & 14.98 & 0.13 & 111.91 & 8.04 & 13.80 & 15.31 & 0.26 & 111.13 & 8.62 \\
\hline & 50 & 13.80 & 64.52 & 0.44 & 101.44 & 0.87 & 13.80 & 64.58 & 0.62 & 101.54 & 1.24 \\
\hline & 200 & 13.80 & 212.91 & 1.63 & 99.55 & 0.81 & 13.80 & 212.72 & 1.63 & 99.45 & 0.81 \\
\hline
\end{tabular}

Table 3. Stability of marker compounds ( $\mathbf{1}-\mathbf{8})$ in $C$. chinensis and C. japonica samples

\begin{tabular}{|c|c|c|c|c|c|c|c|c|c|}
\hline \multirow{2}{*}{ Compound } & \multirow{2}{*}{$\begin{array}{c}\text { Temperature } \\
\left({ }^{\circ} \mathrm{C}\right)\end{array}$} & \multicolumn{6}{|c|}{ Day (\%) } & \multirow{2}{*}{ Mean } & \multirow{2}{*}{$\begin{array}{l}\text { RSD } \\
(\%)\end{array}$} \\
\hline & & 0 & 1 & 3 & 7 & 15 & 30 & & \\
\hline \multirow{2}{*}{1} & 4 & 100 & 100.1 & 101.2 & 101.2 & 97.7 & 99.4 & 99.9 & 1.4 \\
\hline & 25 & 100 & 100.6 & 100.2 & 100.5 & 99.8 & 99.0 & 100.0 & 0.6 \\
\hline \multirow{2}{*}{2} & 4 & 100 & 103.4 & 100.9 & 100.4 & 99.3 & 98.8 & 100.6 & 1.8 \\
\hline & 25 & 100 & 104.4 & 105.8 & 98.6 & 100.5 & 98.7 & 101.6 & 3.3 \\
\hline \multirow{2}{*}{3} & 4 & 100 & 101.2 & 98.9 & 99.6 & 98.4 & 97.1 & 99.04 & 1.5 \\
\hline & 25 & 100 & 97.9 & 98.6 & 99.8 & 100.6 & 98.6 & 99.1 & 1.1 \\
\hline \multirow{2}{*}{4} & 4 & 100 & 100.3 & 100.7 & 98.6 & 96.5 & 98.1 & 98.8 & 1.7 \\
\hline & 25 & 100 & 99.2 & 98.3 & 100.6 & 97.6 & 96.5 & 98.4 & 1.6 \\
\hline \multirow{2}{*}{5} & 4 & 100 & 99.2 & 101.3 & 99.6 & 98.5 & 98.4 & 99.4 & 1.1 \\
\hline & 25 & 100 & 99.6 & 99.0 & 100.2 & 98.5 & 97.0 & 98.7 & 1.2 \\
\hline \multirow{2}{*}{6} & 4 & 100 & 100.1 & 98.1 & 100.7 & 99.9 & 99.6 & 99.7 & 1.0 \\
\hline & 25 & 100 & 100.7 & 99.6 & 98.1 & 100.1 & 99.7 & 99.6 & 0.9 \\
\hline \multirow{2}{*}{7} & 4 & 100 & 100.5 & 99.5 & 99.8 & 99.8 & 99.8 & 99.9 & 0.4 \\
\hline & 25 & 100 & 100.1 & 99.9 & 99.9 & 98.8 & 98.6 & 99.5 & 0.7 \\
\hline \multirow{2}{*}{8} & 4 & 100 & 95.9 & 101.3 & 99.3 & 97.0 & 97.0 & 98.1 & 2.2 \\
\hline & 25 & 100 & 97.5 & 100.7 & 97.2 & 97.6 & 96.4 & 97.9 & 1.6 \\
\hline
\end{tabular}


with the average values of 0.0138 and $0.039 \%$ on dry weight basis, respectively. Meanwhile, compounds 5 - 8 were not shown in their chromatogram (Fig. 2). The peak of compound 3 appeared in the chromatogram of steam processed samples $\mathrm{C} 01-\mathrm{C} 03$. But, it was not detected in that of not-steamed samples C04-C06. The contents of hyperoside in steam processed $C$. chinensis samples $\mathrm{C} 01$ C03 $(0.0153-0.0295 \%)$ were much greater than those of unprocessed samples C04 and C05 (0.0029 and 0.0065\%) (Table 4).

\section{Results and Discussion}

A gradient RP-C18 HPLC system was conducted for simultaneous quantitative determination of eight compounds: hyperoside (1), astragalin, (2), quercetin (3), kaempferol (4), chlorogenic acid (5), 3,4-di- $O$-caffeoylquinic acid (6), 1,5-di-O-caffeoylquinic acid (7), and 4,5di-O-caffeoylquinic acid (8) in extracts from $C$. chinensis and $C$. japonica seeds. Contents of each analyte present in each sample $(C$. chinensis and $C$. japonica) were listed in Table S5. Samples C01-C03 from C. chinensis were steam processed. Two main analytes, compounds $\mathbf{1}$ and $\mathbf{4}$, were in $C$. chinensis samples with average values of 0.0138 and $0.039 \%$ on dry weight basis, respectively. Meanwhile, compounds 5 - 8 were not in their chromatogram. Content of hyperoside in steamed $C$. chinensis samples C01 - C03 (0.0153-0.0295\%) was much greater than those of conventional samples $\mathrm{C} 04$ and $\mathrm{C} 05$ (0.0029 $0.0065 \%)$. A gradient RP-C18 HPLC system was perfor- med for the simultaneous quantitative determination of eight compounds: hyperoside (1), astragalin, (2), quercetin (3), kaempferol (4), chlorogenic acid (5), 3,4-di-Ocaffeoylquinic acid (6), 1,5-di- $O$-caffeoylquinic acid (7), and 4,5-di- $O$-caffeoylquinic acid $(\mathbf{8})$ in the extracts from $C$. chinensis and $C$. japonica seeds.

The contents of each analyte present in each sample $(C$. chinensis and C. japonica) were listed in Table 4. Samples C01 - C03 from C. chinensis were steam processed. Two main analytes, compounds 1 and $\mathbf{4}$, were found in $C$. chinensis samples with the average values of 0.0138 and $0.039 \%$ on dry weight basis, respectively. Meanwhile, compounds 5 - 8 were not shown in their chromatogram (Fig. 2). The content of hyperoside in steam processed samples of C. chinensis C01-C03 (0.0153-0.0295\%) were much greater than those of unprocessed samples C04 and C05 (0.0029 and 0.0065\%) (Table 4). In $C$. japonica samples, compounds $\mathbf{5}$ and $\mathbf{7}$ were major constituents with average values of 0.3274 and $0.2563 \%$ (w/w), respectively. Interestingly, compounds $\mathbf{1}$ - 4 were not revealed in their chromatogram. Therefore, this developed method may be easily and accurately used to distinguish between $C$. chinensis and C. japonica. Cluster analysis of 12 samples was conducted on SPSS software using contents of seven marker components [hyperoside (1), astragalin, (2), quercetin (3), kaempferol (4), chlorogenic acid (5), 3,4-di- $O$-caffeoylquinic acid (6), 1,5-di$O$-caffeoylquinic acid (7), 4,5-di- $O$-caffeoylquinic acid (8)]. Two species of $C$. chienensis Lam. and C. japonica Choisy were unambiguously distinguished by two different

Table 4. The contents (wt\%) of $\mathbf{1}$ - 8 in C. chinensis (C01 - C06) and C. japonica (J07 - J12) samples

\begin{tabular}{|c|c|c|c|c|c|c|c|c|c|}
\hline \multirow{2}{*}{ Samples } & \multicolumn{8}{|c|}{ Compounds } & \multirow{2}{*}{ Species } \\
\hline & 1 & 2 & 3 & 4 & 5 & 6 & 7 & 8 & \\
\hline $\mathrm{CO1}^{\mathrm{a}}$ & 0.0295 & 0.0148 & 0.0034 & 0.0286 & ND & ND & ND & ND & \multirow{7}{*}{$\begin{array}{c}\text { C. chinensis } \\
\text { samples }\end{array}$} \\
\hline $\mathrm{CO2}^{\mathrm{a}}$ & 0.0153 & 0.0171 & 0.0007 & 0.0183 & ND & ND & ND & ND & \\
\hline $\mathrm{C} 03^{\mathrm{a}}$ & 0.0244 & 0.0336 & 0.0028 & 0.0354 & ND & ND & ND & ND & \\
\hline $\mathrm{C04}$ & 0.0029 & 0.1261 & ND & 0.0278 & ND & ND & ND & ND & \\
\hline $\mathrm{CO5}$ & 0.0065 & 0.0340 & ND & 0.0021 & ND & ND & ND & ND & \\
\hline $\mathrm{C06}$ & 0.0045 & 0.0085 & ND & 0.0000 & ND & ND & ND & ND & \\
\hline Average & 0.0138 & 0.039 & 0.0012 & 0.0187 & & & & & \\
\hline J07 & ND & ND & ND & ND & 0.3801 & 0.0422 & 0.3242 & 0.0552 & \multirow{7}{*}{$\begin{array}{c}\text { C. japonica } \\
\text { samples }\end{array}$} \\
\hline J08 & ND & ND & ND & ND & 0.5736 & 0.1770 & 0.4514 & 0.1323 & \\
\hline J09 & ND & ND & ND & ND & 0.1087 & 0.0487 & 0.0315 & 0.0495 & \\
\hline $\mathbf{J 1 0}$ & ND & ND & ND & ND & 0.4317 & 0.1277 & 0.3573 & 0.1103 & \\
\hline J11 & ND & ND & ND & ND & 0.1230 & 0.0501 & 0.0551 & 0.0591 & \\
\hline J12 & ND & ND & ND & ND & 0.3474 & 0.0730 & 0.3183 & 0.0655 & \\
\hline Average & & & & & 0.3274 & 0.0864 & 0.2563 & 0.0786 & \\
\hline
\end{tabular}

${ }^{\mathrm{a}} \mathrm{C} 01$ - $\mathrm{C} 03$ samples were steam processed.

ND: not detect. 


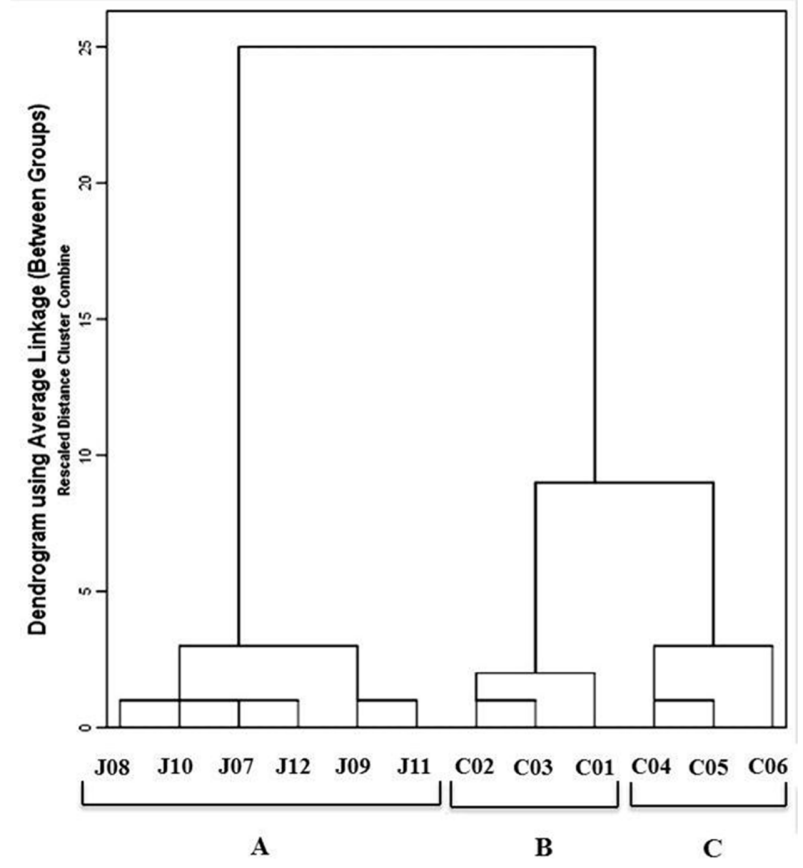

Fig. 3. The cluster analysis of $C$. chinensis and $C$. japonica seed samples using the contents of eight marker compounds $(\mathbf{1}-\mathbf{8}) . C$. japonica seed samples (J07 - J12, A), C. chinensis steam processed $(\mathrm{C} 01-\mathrm{C} 03, \mathrm{~B})$ and $C$. chinensis unprocessed seed samples (C04 - C06, C).

groups. In addition, two clusters of $C$. chinensis steam processed (C01-C03) and unprocessed samples (C04C06) were also separated by the dendrogram (Fig. 3).

In conclusion, eight marker compounds $(\mathbf{1}-\mathbf{8})$ were identified in the $C$. chinensis and C. japonica samples. Particularly, development of the reliable HPLC-PDA method simultaneously quantitate eight marker compounds [hyperoside (1), astragalin, (2), quercetin (3), kaempferol (4), chlorogenic acid (5), 3,4-di-O-caffeoylquinic acid (6), 1,5-di-O-caffeoylquinic acid (7), 4,5-di-O-caffeoylquinic acid luteolin (8), an internal standard (caffeic acid). Results indicated that the method was applied for the quality evaluation of seeds between the $C$. chinensis and C. japonica.

\section{Acknowledgments}

This work was supported by the sabbatical research grant from Daegu Catholic University in 2018.

Conflict of Interest - The authors declare no conflict of interest.

\section{References}

(1) Donnapee, S.; Li, J.; Yang, X.; Ge, A. H.; Donkor, P. O.; Gao, X. M.; Chang, Y. X. J. Ethnopharmacol. 2014, 157, 292-308.

(2) He, X. H.; Yang, W. Z.; Meng, A. H.; He, W. N.; Guo, D. A.; Ye, M. J. Asian. Nat. Prod. Res. 2010, 12, 934-939.

(3) Sam, H. V.; Baas, P.; Kebler, P. J. A. Blumea 2008, 53, 569-601.

(4) Zhen, G. H.; Jiang, B.; Bao, Y. M.; Li, D. X.; An, L. J. J. Chin. Med. Mat. 2006, 29, 1051-1055.

(5) Liu, J.; Jiang, B.; Bao, Y.; An, L. Int. J. Dev. Neurosci. 2003, 21, 277-281.

(6) Yen, F. L.; Wu, T. H.; Lin, L. T.; Lin, C. C. J. Ethnopharmacol. 2007, 111, 123-128.

(7) Yao, C. H.; Tsai, H. M.; Chen, Y. S.; Liu, B. S. J. Biomed. Mater. Res. B. Appl. Biomater. 2005, 75B, 277-288.

(8) Chauhan, A.; Sharma, P. K.; Srivastava, P.; Kumar, N.; Dudhe, R. Pharm. Lett. 2010, 2, 369-387.

(9) Cheng, P.; Shi, J.; Du, P.; Liu, D.; Cao, X.; Wen, X. Academic Periodical of Farm Products Processing. 2013, 8, 116-118.

(10) Liao, G. I.; Chen, M. Y.; Kuoh, C. S. Bot. Bull. Acad. Sin. 2005, 46, 75-81.

(11) Araujo P. J. Chromatogr. B Analyt. Technol. Biomed. Life Sci. 2009, 877, 2224-2234.

(12) Le, D. D.; Nguyen, D. H.; Zhao, B. T.; Min, B. S.; Song, S. W.; Woo, M. H. Nat. Prod. Sci. 2019, 25, 122-129.

(13) Hajimehdipoor, H.; kondori, B. M.; Amin, G. R.; Adib, N.; Rastegar, H.; Shekarchi, M. Daru. 2012, 20, 57-63.

Received July 18, 2019

Revised October 24, 2019 Accepted October 25, 2019 